\title{
EL PAPEL DE LOS DICCIONARIOS PEDAGÓGICOS ESPECIALIZADOS EN LA ENSEÑANZA/APRENDIZAJE DEL ESP
}

\author{
Pedro A. Fuertes-Olivera \\ Universidad de Valladolid \\ pedro@tita-emp.uva.es
}

Beatriz Pérez Cabello de Alba

UNED. Madrid

bperez-cabello@flog.uned.es

\section{RESUMEN}

Con el nombre de Inglés para Fines Específicos, mas conocido por el acrónimo inglés ESP, nos referimos a la variedad de la lengua inglesa que se enseña a los estudiantes matriculados en carreras como Económicas, Turismo, Medicina e Ingenierías. Desde sus inicios hasta la fecha hemos asistido a un gran crecimiento en el número de estudiantes y cursos ofertados, que ha obligado a poner en marcha prácticas docentes autónomas, es decir sin la participación de expertos en el campo, y replicativas, es decir prácticas que fueron inicialmente diseñadas para el aprendizaje de las destrezas profesionales, como por ejemplo el uso del estudio de casos procedente de los Masters of Business Administration (MBA). Ambos procesos, unidos al desarrollo del inglés como lingua franca y al interés por el desarrollo de una metodología que favorezca la autonomía de los estudiantes, se han traducido en un desarrollo espectacular de herramientas docentes de Internet. En este marco se sitúa este trabajo que se organiza en tres grandes apartados: (1) una precisión del concepto de ESP y una reinterpretación del mismo; (2) una revisión del concepto de lexicografía y del diccionario pedagógico; (3) una descripción de las 
posibilidades que ofrecen los diccionarios de Internet para poder leer textos de inglés empresarial/inglés económico. El artículo finaliza resumiendo las principales ideas defendidas en este trabajo.

Palabras Clave: ESP; diccionario de Internet; autonomía; enseñanza/aprendizaje, lectura/recepción.

\section{ABSTRACT}

From its inception in the early 1960s, English for Specific Purposes (ESP) has grown to become one of the most prominent areas of EFL teaching. ESP is usually concerned with the teaching of English used in academic studies and/or professional activities, especially the teaching of English related with Business/Economics, Tourism, Medicine, and Engineering. As the number of courses and students has grown considerably the original team teaching methodology is being substituted by a more autonomous approach in which both ESP instructors and students have to acquire knowledge of the basic concepts of the field by themselves, as well as upgrade their discourse competence. This new approach lends support to recent developments in the field of learner's autonomy, especially those based on Internet (for example, Moodle) that are being promoted in this era of shortages and lack of resources. Within this framework, this article offers a review of the state of art regarding ESP, formulates a reconceptualization of the concept with the aim of making room to two recent developments: the consolidation of English as lingua franca; and the possibilities offered by the Internet for teaching and learning. It also enumerates the main basic requirements of pedagogically-oriented specialised dictionaries and illustrates some of their potentialities for assisting users in a reading situation. The article finishes by summarising the main conclusions drawn.

KEY WORDS: ESP; Internet dictionary; learner's autonomy; teaching/learning; reading/reception

\section{El Inglés PARA Fines EsPecíficos (ESP)}

La investigación sociolingüística ha puesto de manifiesto que ninguna sociedad tiene una estructura lingüística uniforme y estática. Existen dialectos de naturaleza diversa, como los que conocemos como sublenguajes, que son registros o variedades lingüísticas que tienden hacia la mono-referencialidad con el fin de eliminar la ambigüedad y la polisemia. Estos registros, a los que nos referimos con el nombre genérico de lenguajes de especialidad, obligan a sus usuarios potenciales a realizar un aprendizaje consciente de los mismos, algo que se traduce en la existencia de un número restringido de usuarios que están obligados a aprender una temática específica gracias a la cual podrán comunicarse en un marco restringido de comunicación.

Uno de los lenguajes de especialidad más estudiados es el inglés especializado, que empieza su andadura académica a finales de la década de 1960. Desde entonces, el estudio del inglés especializado ha ganado presencia en la mayoría de las universidades del mundo pudiendo observar dos líneas de trabajo bien delimitadas: una de estas líneas 
prioriza la investigación terminológica y traductológica (y se lleva a cabo fundamentalmente en departamentos y facultades de comunicación y traducción, al menos en España), mientras que la otra se centra en los desafíos que esta variedad presenta en el aula. A esta segunda línea de trabajo se la conoce con el nombre genérico de English for Specific Purposes (ESP).

El ESP por lo tanto trata de ofrecer soluciones específicas a necesidades específicas, algunas de las cuales se han identificado en un marco concreto mediante enfoques cuantitativos y cualitativos. Por ejemplo se afirma que el proceso de enseñanza/aprendizaje del Inglés Empresarial (ESPB en inglés) está muy relacionado con la influencia de los factores de aprendizaje (Skehan, 1989) y con el conocimiento de las potencialidades que ofrecen los géneros como mecanismos comunicativos contextualizados, que son objeto de enseñanza combinando destrezas lingüísticas y profesionales. Por ejemplo, las actividades orales como «negociaciones» y «reuniones» forman la parte central de la mayoría de los programas dedicados a la mejora de las destrezas comunicativas orales de los estudiantes de un curso de ESPB partiendo de la idea que señala que una de las características básicas de toda negociación o reunión es el diseño de una estrategia que permita, de alguna forma, establecer algún tipo de complicidad con la otra parte. Igualmente, las actividades escritas, principalmente los correos electrónicos y las cartas comerciales, van a desarrollarse partiendo del concepto move que va a estar sometido a un proceso de reactualización permanente para adecuarlo a situaciones novedosas como son la existencia de diferencias culturales (en el aula y fuera del aula) y la familiaridad con las tecnologías colaborativas que Internet hace posible (Fuertes-Olivera y Pérez Cabello de Alba, 2012).

La idea dominante en el contexto que acabamos de señalar es el abandono de una práctica que ha gozado de gran aceptación en el campo de enseñanza/aprendizaje de una lengua extranjera: debemos dejar a un lado el objetivo de la competencia (proficiency) entendida como un intento por lograr que nuestros estudiantes se comporten lingüísticamente de la forma más parecida posible a como lo haría un hablante nativo y centrarnos en la enseñanza de las estrategias retóricas y las destrezas profesionales incluyendo sus manifestaciones lingüísticas tal y como se manifiestan en los diferentes géneros que conforman el ESP en general y cada una de sus manifestaciones en particular. Es en este marco donde situamos este trabajo que propone una reconceptualización del concepto de ESP (sección 2) que facilite la mejora de la cultura lexicográfica de los estudiantes y participantes en un curso de ESP y el empleo de diccionarios como herramientas pedagógicas (sección 3), como por ejemplo los diccionarios de Internet utilizados para ayudar al estudiante a mejorar la recepción de textos económicos/ empresariales (sección 4). El trabajo finaliza resumiendo las principales conclusiones alcanzadas.

\section{UNA VISIÓN DIFERENTE DEL ESP}

El concepto tradicional de ESP, por ejemplo la práctica docente llevada a cabo por un profesor de inglés y un especialista, debe modificarse ligeramente para dar cabida a una realidad nueva caracterizada por la falta de recursos para poder atender al desarrollo exponencial del ESP debido a la consolidación del inglés como lingua franca de la comunicación especializada (Ljosland, 2011). Este hecho es fácilmente observable en el campo 
del Business English, es decir el inglés descrito como un híbrido que mezcla contenido general y especializado y tiene como objetivo ayudar a sus aprendices a adaptar/adoptar los procedimientos y convenciones empresariales, hacer un uso selectivo de los recursos léxico-gramaticales de la lengua inglesa y utilizar los recursos de audio y video gracias a los cuales podamos replicar en las clase de ESP las metodologías y procedimientos empleados en los cursos de MBA (por ejemplo, los case studies) con vistas a lograr un manejo razonable de la modalidad escrita, hablada, e incluso de la multimodalidad (Zhang, 2007).

La consolidación de una lingua franca tan dominante como el inglés tiene tres consecuencias para el proceso de enseñanza/aprendizaje: no podemos esperar que todos los estudiantes de ESP adquieran un manejo de la lengua inglesa como si fueran hablantes nativos; no podemos esperar que existan recursos suficientes que permitan enseñar gracias al esfuerzo conjunto de un profesor de inglés y un especialista; y no podemos esperar que las variedades dominantes del inglés, es decir el British English y el American English, sigan ejerciendo el mismo dominio que han ejercido hasta la fecha. Por ejemplo, la investigación actual señala la existencia de millones de «hablantes» de la lengua inglesa cuyo interlenguaje plantea problemas comunicativos tanto a los hablantes nativos como a otro tipo de hablantes (Rogerson-Rovell, 2008).

Una manera de solucionar la situación que hemos descrito en el párrafo anterior es proponiendo una práctica docente que diferencie entre el inglés general especializado y el inglés solo especializado (Fuertes-Olivera, 2011). El primero, que es al que nos vamos a referir en este trabajo, es un lenguaje o discurso especializado lo más general posible que normalmente va destinado al semi-experto estudiante, es decir al usuario que está en camino de convertirse en un experto.

El aprendizaje del inglés general especializado puede conseguirse mediante el uso combinado de tres enfoques que suelen presentarse por separado en el campo del ESP: el enfoque funcional, el enfoque estilístico y el enfoque léxico. El enfoque funcional defiende que el lenguaje es un sistema comunicativo que utiliza una serie de recursos expresivos que sirven a una finalidad determinada. Este enfoque se ocupa, básicamente, del análisis de las funciones del lenguaje, de las diferencias entre textos especializados orales o escritos, y de establecer una tipología textual de textos especializados conforme a una serie de criterios, como son por ejemplo el ámbito de la comunicación y la temática, el nivel de especialización, el canal de comunicación y la forma estilística empleada en el tratamiento del tema. Los partidarios de este enfoque creen que el lenguaje de especialidad es un uso restringido del lenguaje general en el que lo importante es el registro, es decir la variedad de una lengua de acuerdo a la situación en la que aparece, que tiene que ver con las diferentes maneras de construir textos. Dentro de este marco general destacan los trabajos que promueven el trabajo docente con textos híbridos, como por ejemplo trabajos que ponen de manifiesto las diferencias entre registros atribuidas no solo al mismo registro sino también a que el autor sea nativo o no nativo, a que el autor pertenezca a una u otra tradición cultural, o a que vayan destinados a un usuario experto o semi-experto (Koyalan y Mumford, 2011). En resumen, en un programa de inglés general especializado el enfoque funcional ofrece argumentos teóricos para conseguir que el estudiante maneje las funciones generales destinadas a facilitar la comunicación.

El enfoque estilístico tiene una relación muy estrecha con el enfoque anterior, aunque es diferente al prestar más atención al concepto estilo que al concepto regis- 
tro. Los partidarios de este enfoque defienden que en el discurso especializado puede observarse la presencia de un conjunto de recursos verbales y no verbales que facilitan la consecución de un objetivo comunicativo concreto, en unas condiciones específicas determinadas, por los miembros de la sociedad. Es posible, por ejemplo, referirnos al estilo científico, nombre con el que suele describirse un texto escrito de forma objetiva, lógica, precisa, abstracta, y exacta en el que predominan las construcciones pasivas, las oraciones complejas, las nominalizaciones, las construcciones subordinadas, etc. (Bathia, 2008; Samaniego Fernández, 2007). En resumen, el enfoque estilístico nos ayudará en un programa de inglés general especializado proponiendo la enseñanza/aprendizaje de los elementos más abstractos comunes a todos los estilos agrupados bajo el marco común del lenguaje especializado.

Finalmente, el enfoque léxico afirma que el léxico es el elemento diferenciador más importante del discurso especializado y por ello promueve una enseñanza centrada en precisar los términos y semi-términos de cada campo y subcampos. Este enfoque sustenta, entre otras cosas, la construcción de diccionarios pedagógicos y el desarrollo de una cultura lexicográfica con la que se espera mejorar la competencia léxica del aprendiz (Fuertes-Olivera y Pérez Cabello de Alba, 2012). Este enfoque nos ayudará en un programa de inglés general especializado mejorando la cultura lexicográfica/terminológica de los participantes en el curso, como vamos a ver en las secciones 3 y 4 de este artículo.

La combinación de los tres enfoques nos permite llevar a cabo una reconceptualización del ESP gracias a la cual podamos mantener las características tradicionales básicas del concepto con la situación social marcada por el dominio del inglés como lingua franca. Las características tradicionales las resumimos en la idea que mantiene que el ESP es un movimiento académico ocupado en el diseño de enfoques, métodos y procedimientos docentes con los que lograr satisfacer las necesidades comunicativas que un grupo de estudiantes específicos tienen o puedan tener en una situación de uso específica. En términos generales podemos afirmar que esta idea es similar a la que sustenta la mayoría de los enfoques comunicativos (Munby, 1978). Por ejemplo, si el curso de ESP está planteado para preparar a los estudiantes a escribir cartas comerciales, el enfoque, el método y el procedimiento docente tienen que traducirse en una práctica docente que incluya los modelos de carta posibles, las diferencias culturales, el uso en contexto de las principales estructuras, y la práctica real, es decir escribir cartas que reproduzcan situaciones reales. En resumen, el mantenimiento de los aspectos tradicionales incluidos en nuestra reconceptualización del concepto de ESP se orientan a mantener el carácter especializado de la situación y del usuario potencial.

Por otra parte defendemos la inclusión de aspectos novedosos que tengan en cuenta una situación dominada por el inglés como lingua franca y por la aparición de Internet. En este marco proponemos una reconceptualización del ESP que nos permite afirmar que en la era de Internet el ESP es fundamentalmente un proceso de enseñanza/aprendizaje en marcha continua, en el que participan seres humanos que pueden comunicarse presencialmente y no presencialmente, que pueden o no pueden formar parte de una misma cultura, que normalmente son capaces de identificar sus necesidades comunicativas específicas, y que necesitan compaginar y hacer compatible el dominio de conocimiento experto y competencia discursiva mediante una práctica docente que se construye combinando los aspectos fundamentales del enfoque funcional, estilístico y léxico. En otras 
palabras, nuestra reconceptualización del concepto de ESP incide en su naturaleza como lengua instrumental de comunicación, normalmente utilizada con y por hablantes nativos y no nativos, que deben prescindir de todo aquello que pueda dificultar la comunicación (por ejemplo, el empleo de fórmulas muy idiomáticas) pero que deben hacer uso de todos los recursos disponibles que puedan mejorar su competencia comunicativa especializada, aquí definida como la capacidad de comunicarse con otro experto o aspirante a serlo con independencia de que haya entre ellos afinidades culturales, lingüísticas o geográficas. De acuerdo con esta idea la competencia comunicativa especializada debe aspirar a transmitir mensajes claros y diáfanos que no dificulten la consecución del conocimiento experto, que es la base sobre la que se asienta cualquier intento de comunicación entre especialistas, aspirantes a serlo, o personas interesadas en el mismo.

En trabajos anteriores (Fuertes-Olivera y Pérez Cabello de Alba, 2012) nos hicimos eco de las posibilidades que ofrece Internet para poder mejorar nuestro conocimiento experto en una situación comunicativa en la que el team teaching no existe o no es posible esperarlo de una manera realista. En este trabajo seguimos en esta línea, aunque restringida a un aspecto o destreza comunicativa: la recepción de textos especializados, quizás una de las destrezas que puedan conseguirse con un coste aceptable en el marco de la universidad española actual en donde no es fácil mejorar otras destrezas de los estudiantes teniendo en cuenta las lagunas conceptuales y comunicativas que muestran la mayoría de los estudiantes universitarios españoles (Fuertes-Olivera, 2009 y 2012).

\section{El DICCIONARIO COMO HERRAMIENTA PEDAGÓGICA}

La investigación actual lexicográfica señala que la lexicografía es un disciplina científica independiente que se ocupa de la teoría y práctica necesarias para compilar herramientas de uso caracterizadas por facilitar un acceso rápido y fácil a los datos lexicográficamente relevantes de los cuales un tipo específico de usuario pueda extraer el tipo específico de información que le permita satisfacer el tipo específico de necesidades (lexicográficamente relevantes) que tenga en un tipo específico de situación social extralexicográfica, o sea, independientemente del uso o no que pueda hacerse del diccionario (Bergenholtz y Tarp 2002, 2003; Tarp 2008).

La reflexión que acabamos de reproducir tiene también aplicaciones prácticas, y nos obliga a interrogarnos sobre las características que debe tener un diccionario concebido como herramienta pedagógica. Por ejemplo un diccionario cuyas entradas reproducimos en el ejemplo (1) puede llegar a ser nocivo desde el punto de vista pedagógico:

delinquent (adj. y n.). Delincuente, moroso.

d. (overdue, past-due) account. Crédito vencido hace tiempo, cuenta morosa.

d. list. Lista de morosos, listado de morosos [Hisp.].

d. payments. Morosidad en el pago.

d. return. Declaración morosa.

d. tax. Impuesto atrasado, pendiente de liquidación. Impuesto en mora.

d. taxpayer. Contribuyente moroso.

delincuente (p.a. y s.). Delinquent. Criminal. Culprit. Offender. Defaulter. Guilty. Defaulter (soldier). 
moroso, sa (adj.) Slow. Delinquent (in payment). Tardy. Heavy. Laggard. Morose. Debtor in arrears. Defaulter. Slow pay.

Contribuyente moroso: Delinquent taxpayer.

Cuenta morosa: Delinquent account.

Declaración morosa: Delinquent return.

Deudor moroso: Bad (tardy) debt. Defaulting debtor. Unsafe debtor.

Lista de morosos: Delinquent list.

Ejemplo 1: Entradas en Pirámide Economía y Empresa 2005.

Para un aprendiz de ESP, la entradas del ejemplo 1 tienen varios problemas: (a) errores conceptuales, es decir errores relacionados con el conocimiento experto como mantener que «delincuente» y «moroso» son sinónimos en español comercial; (b) errores relacionados con el manejo comunicativo como el empleo de códigos gramaticales confusos y contradictorios (por ejemplo usan «n» $\mathrm{y}$ «s» para referirse a la misma clase de palabra), y (c) la falta de precisión del significado de la entrada y su equivalente. En resumen la consulta de un diccionario con entradas como las del ejemplo 1 no es aconsejable desde un punto de vista pedagógico.

Por el contrario, un participante en un proceso de enseñanza/aprendizaje de ESP verá facilitada su labor si busca ayuda en un diccionario pedagógico especializado, es decir en un diccionario que se ha confeccionado para ayudar a los aprendices de una lengua extranjera que cursan una carrera como Económicas (por razones de espacio nos vamos a limitar en lo que resta de trabajo al ESPB) y que por lo tanto va destinado a un aprendiz de la Economía y de su discurso.

Revisamos a continuación las características o requisitos de ese diccionario para estudiantes de ESP partiendo para ello de algunos de los principios aceptados en este campo del saber, que pueden estudiarse con más detalle en la literatura especializada, por ejemplo en Bogaards (1996), Fuertes-Olivera (2010a y 2012) Fuertes-Olivera y Arribas-Baño (2008), Kaalep y Mikk (2008), Pujol, Corrius y Masnou (2006), Rundell (1999), Scholfield (1999) Tarp (2004) y (2008), y Verlinde y Binon (2009). Por motivos de espacio vamos a referirnos a tres requisitos básicos y generales que afectan a todos los tipos de diccionarios pedagógicos. Son criterios muy generales que, si se tienen en cuenta, serán de gran ayuda a la hora de sacar un mayor rendimiento al empleo de un diccionario en una clase de ESP.

El primero de los requisitos tiene que ver con la situación general de uso y las características del aprendiz, es decir con la situación de aprendizaje (learning situation) y las necesidades del usuario (user's needs). Ambas características nos obligan a tener en cuenta variables como nivel de dominio de la lengua del usuario, edad del usuario, características socioeconómicas del aprendiz (por ejemplo, que el aprendiz sea un emigrante es muy diferente a que sea un estudiante nacional), afinidad cultural, nivel cultural, la participación del aprendiz en un proceso de aprendizaje consciente o inconsciente, y que el proceso ocurra en un lugar donde se habla la lengua que se estudia o no. Todas estas variables tienen influencia lexicográfica y por ello determinan la calidad pedagógica del diccionario y su usabilidad en una situación de aprendizaje concreta (Fuertes-Olivera, 2012). Cada variable que acabamos de mencionar incide sobre el carácter pedagógico del diccionario. Por ejemplo, el nivel de dominio de la lengua influye en el tipo de diccionario recomendable. 
Si un usuario es un principiante (beginner) no puede usar un diccionario monolingüe como el Cobuild (Sinclair, 1987) ya que este diccionario está destinado a estudiantes de nivel avanzado. Igualmente, si el aprendiz está en contacto diario y permanente con la lengua que quiere aprender necesitará diccionarios con muchos lemas y diccionarios actualizados ya que en ese contexto está expuesto a la fuerza creativa de cada lengua. Por el contrario, si está en un contexto de aprendizaje artificial, es decir en un lugar en donde la lengua que estudia no es de uso diario, puede usar diccionarios con menos lemas y más antiguos. Por ejemplo, para un español que esté aprendiendo el inglés en el Reino Unido el Gran Diccionario Oxford de 2008 (Rollin y Styles Carvajal, 2008) es más recomendable que el Collins Inglés-Español (Smith, 1987) porque el primero tiene más entradas y frases (300.000) que el segundo (200.000) y porque el primero recoge más palabras «nuevas», es decir palabras que acaban de crearse y acaban de entrar en el sistema y a las que dedica un tratamiento lexicográfico bastante adecuado. Por ejemplo en la edición digital del 16 de febrero de 2012, el tabloide The Sun incluye un artículo sobre los temores de la familia de Whitney Houston por el comportamiento que pudiera tener su exmarido. En el texto aparecen un buen numero de palabras y expresiones coloquiales; son elementos léxicos que nos indican la riqueza expresiva de la lengua y su capacidad de creación constante. Son además elementos muy usados por los hablantes nativos y, como veremos en el cuadro 1, su tratamiento lexicográfico en los dos diccionarios antes mencionados reafirma la posición que acabamos de mantener: un diccionario con más lemas y más actualizado ofrece un tratamiento más adecuado que un diccionario con menos lemas y más antiguo. Este punto es especialmente relevante cuando el aprendiz desea manejar la lengua oral y actual, que es una de las características de la lengua informal propia del registro oral (cuadro 1).

Cuadro 1. Equivalentes de palabras inglesas informales en el Gran Diccionario Oxford y en el Collin Inglés-Español

\begin{tabular}{|c|c|c|}
\hline $\begin{array}{l}\text { Palabra } \\
\text { inglesa }\end{array}$ & Equivalente en Gran Diccionario Oxford (resumen) & $\begin{array}{c}\text { Equivalente en Collins } \\
\text { Inglés-Español } \\
\text { (resumen) }\end{array}$ \\
\hline showbiz & $\begin{array}{l}\text { (colloq) mundo } m \text { del espectáculo, farándula } f \text { (period), } \\
\text { (before } \mathrm{n}) \text { (personality/news) del mundo del espectáculo }\end{array}$ & V. show business \\
\hline mum & $\begin{array}{l}\text { A. (mother) (BrE colloq) mamá (fam) (as form of } \\
\text { address) mamá } \\
\text { B. (silence) (colloq) mum's the word punto en boca! } \\
\text { (fam) chitón! (fam); to keep mum no decir ni pío (fam) }\end{array}$ & (British) mamáf \\
\hline ban & $\begin{array}{l}\text { (book/smoking) prohibir; (organization) proscribir; } \\
\text { (activity) prohibir, vedar (...) }\end{array}$ & $\begin{array}{l}\text { prohibir, proscribir; person } \\
\text { excluir }(. . .)\end{array}$ \\
\hline bad blood & resentimiento $m$, animosidad $f$ & mala $f$ leche, mala $f$ uva \\
\hline build bridges & tender un puente (de unión) & \\
\hline $\begin{array}{l}\text { fall off the } \\
\text { wagon }\end{array}$ & dejar de beber & no beber \\
\hline crave for & tener ansias de & ansiar, anhelar \\
\hline alias & alias & $\begin{array}{l}\text { alias } m \text {, nombre } m \text { ficticio, } \\
\text { seudónimo } m\end{array}$ \\
\hline pal & (friend) (colloq) amigo $m$, compinche $m$ (fam) & camarada $f$ compinche $m$ \\
\hline say farewells & $\begin{array}{l}\text { Despedirse de alguien o de algo, decirle adiós a alguien/ } \\
\text { algo }\end{array}$ & \\
\hline
\end{tabular}


El segundo requisito se refiere al objeto de aprendizaje, es decir a la naturaleza de lo que estamos estudiando. Este requisito nos obliga a prestar atención al nivel de dominio del usuario (por ejemplo que sea un experto o no lo sea), es decir nos fuerza a analizar la cantidad y calidad de la información enciclopédica que debe tener el diccionario así como el empleo de mecanismos de precisión del significado del término. Por ejemplo, si un estudiante necesita adquirir conocimiento experto no debe nunca usar un diccionario de lengua general ya que estos diccionarios no suelen precisar los significados de los términos. Por ejemplo el Gran Diccionario Oxford (Rollin y Styles Carvajal, 2008) ofrece dos significados de accounting (contabilidad y teneduría de libros). Uno de los significados (teneduría de libros) es erróneo desde el punto de vista conceptual, mientras que el otro (contabilidad) es impreciso. El término inglés accounting realmente significa dos realidades conceptuales próximas que un diccionario bien concebido debe recoger, como el Diccionario InglésEspañol de Contabilidad: Recepción (Fuertes-Olivera y otros, 2012) (ejemplo 2):

Definición de contabilidad:

1. La contabilidad comprende el conjunto de actividades profesionales llevadas a cabo por los contables, como son la preparación de las cuentas anuales, su registro y la preparación de los registros financieros de acuerdo con las disposiciones fiscales.

2. La contabilidad es el proceso de contabilización incluyendo la identificación, medición y anotación de las transacciones de una empresa y comprende la contabilidad financiera y la contabilidad de gestión. La contabilidad financiera es la comunicación de la información financiera a los usuarios externos y comprende el registro de los datos financieros en unidades monetarias anotadas en los estados financieros, como son el balance, la cuenta de pérdidas y ganancias y el estado de flujos de efectivo. La contabilidad gerencial es para uso interno por parte de la dirección de una empresa.

Ejemplo 2: Entradas en el Diccionario Inglés-Español de Contabilidad: Recepción (Fuertes-Olivera y otros, 2012).

Las definiciones del ejemplo 2 son adecuadas desde el punto de vista pedagógico porque ayudan a los usuarios potenciales ofreciendo una información precisa (es decir, permiten adquirir conocimiento experto) y están en consonancia con la naturaleza del discurso especializado en el que la exactitud y precisión conceptual son completamente necesarias. Es decir, es una entrada pensada para ofrecer conocimiento experto y favorecer la adquisición de la competencia comunicativa, por ejemplo para leer un texto de contabilidad.

El tercero de los requisitos está relacionado con la calidad lexicográfica del diccionario, es decir con las estructuras lexicográficas del diccionario. Bogaards (1996) afirma que la calidad pedagógica del diccionario depende de tres grandes parámetros conocidos como encontrabilidad (findability), comprensibilidad (comprehensibility) y usabilidad (usability). La encontrabilidad es la facilidad para encontrar lo que uno busca. En un diccionario la encontrabilidad está relacionada con el número de lemas y expresiones que tiene y con la forma y manera de acceder a los datos lexicográficos. Este criterio ha sido objeto de una gran atención en los últimos años y podemos observar diferencias notables entre los diccionarios que han prestado atención a este concepto y los que no. Por ejemplo, en el Macmillan English Dictionary for Advanced Learners (Rundell, 2007) es mucho más fácil encontrar una palabra o expresión que en el Oxford Advanced Learner»s Dictionary (Cowie, 1989). La diferencia fundamental entre ambos diccionarios es el empleo de me- 
canismos o estructuras lexicográficas pensadas para facilitar la búsqueda. Por ejemplo el verbo get en el Macmillan va introducido por un índice con 15 significados diferentes seguido por «phrases» $\mathrm{y}$ «phrasal verbs». Consultando el índice el usuario puede ir directamente al significado que puede necesitar. Nada de esto aparece en el Oxford por lo que el usuario tiene que leer toda la entrada (casi cuatro páginas) hasta encontrar lo que busca.

La comprensibilidad está conectada con el empleo de mecanismos lexicográficos que facilitan la comprensión del significado, especialmente el uso de definiciones sencillas, y mecanismos de desambiguación del significado como ejemplos de uso, ilustraciones, etc. Esta variable es especialmente necesaria en el discurso especializado y, como ya dijimos al comentar el ejemplo 1 , debe restringirse el uso de los diccionarios que no ofrecen mecanismos de desambiguación. Por ejemplo, el Diccionario LID Empresa y Economía (Elosúa, 2007) solo ofrece una definición de cada término y un equivalente en varias lenguas (inglés, francés, alemán y portugués). La definición es compleja y solo puede ser útil en un marco conceptual restringido a expertos (ejemplo 3). En cambio, la misma entrada en un diccionario pedagógico como el Oxford Business English (Parkinson, 2005) es adecuada ya que la definición es más sencilla y va acompañada de información gráfica, datos gramaticales, ejemplos, etc. que precisan el significado (ejemplo 4).

\section{Matriz de crecimiento-cuota}

Matriz diseñada por The Boston Consulting Group para clasificar los negocios de un grupo de empresas en función del crecimiento esperado del segmento (más o menos de una media, por ejemplo el 5\% real) y de la cuota de mercado del negocio en ese segmento (dominante o no). El calificativo de vaca, estrella, interrogación o perro facilita una comprensión superficial de la misión de cada negocio.

Ejemplo 3: Entrada en el Diccionario LID Empresa y Economía

The Boston Matrix / 'bostən; AmE 'bo:s-/ (also, growth-share matrix) noun [C, usually sing] (Marketing) a way of analysing how successful a range of company's products or services are by looking at the percentage of sales it has in the market and how fast the sales are growing

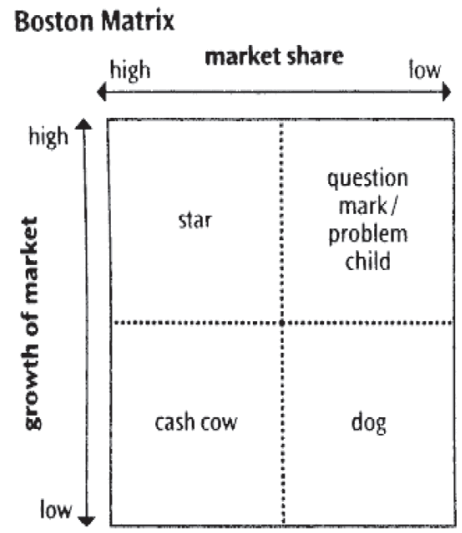

Ejemplo 4: Entrada en el Oxford Business English 2005 
Finalmente, el criterio de usabilidad está relacionado con las funciones del diccionario, es decir con el uso específico del diccionario o bien para ayudarnos a adquirir los conceptos del campo o las características de su discurso, o ambas cosas a la vez. Veremos a continuación un ejemplo de usabilidad al analizar el empleo de los diccionarios de Internet para leer (es decir recibir) textos de inglés económico.

\section{LOS DiCCIONARIOS DE INTERNET Y LA RECEPCIÓN DE TEXTOS EN INGLÉS ECONÓMICO}

Un diccionario de Internet es un repositorio lexicográfico construido para ser consultado a través de la red. Hay tres tipos básicos de diccionarios de Internet: (i) un diccionario impreso que se sube a la red como pdf; (b) un diccionario estático, es decir un diccionario que no permite interactuar ni realizar búsquedas diferentes a usuarios diferentes en situaciones de uso diferentes; (c) un diccionario dinámico, que es un diccionario construido de acuerdo con las posibilidades de Internet permitiendo búsquedas customizadas y facilitando la interacción entre el diccionario y el usuario (en FuertesOlivera y Bergenholtz, 2011 se puede encontrar información sobre el estado actual de la lexicografía de Internet).

En trabajos anteriores (Fuertes-Olivera, 2009; Fuertes-Olivera 2010b; FuertesOlivera, 2012; Fuertes-Olivera y Nielsen, 2011 y 2012; Fuertes-Olivera y Pérez Cabello de Alba, 2012) hemos señalado que los diccionarios estáticos son muy abundantes en Internet y, además, suelen ser de acceso libre y, por tanto, gratuitos. Estos diccionarios ofrecen datos lexicográficos similares a los que encontramos en los diccionarios impresos y pueden por ello usarse en una situación extra-lexicográfica típica: alguien, por ejemplo un estudiante de ESPB desea ayuda para leer un texto de economía/empresa. En este marco ese usuario se beneficiará del uso de un diccionario de Internet si, además de tener en cuenta las características comentadas en la sección anterior, presta atención a dos elementos específicos que desarrollaremos a continuación: la naturaleza del discurso económico, y la relación entre diccionario pedagógico y recepción de un texto.

El discurso económico/empresarial destaca por su naturaleza híbrida, es decir por conjugar características propias del discurso general y de cada uno de sus subdominios. Este hecho influye en la cultura lexicográfica del usuario al obligarle a decidir entre diccionarios especializados generales y diccionarios de subespecialidad (Fuertes-Olivera, 2012; Fuertes-Olivera y Nielsen, 2011 y 2012). Los primeros son diccionarios como el Oxford Business English (Parkinson, 2005) que cubren todos lo subcampos del dominio empresa/economía, aunque de una manera superficial. Los segundos son diccionarios de subespecialidad que se centran en el léxico especializado de un subcampo, por ejemplo, «marketing», «contabilidad», «finanzas», y son por tanto mucho más específicos y ofrecen un tratamiento más detallado de los términos. Los diccionarios especializados generales son adecuados para recibir textos generales de naturaleza económica, es decir textos que tratan de economía/empresa sin ofrecer detalles específicos.

Los textos generales de naturaleza económica son textos que suelen publicarse en periódicos y revistas especializadas como Financial Times, Wall Street Journal y The Economist. Muchos profesores en España utilizan textos de estos periódicos y revistas para mejorar la comprensión lectora de sus estudiantes. Por ejemplo el texto «A darkening mood» 
(edición digital del 14 de febrero de 2012 de The Economist) podría ser usado para mejorar la competencia lectora. En este texto hay algunos términos que son imprescindibles para entenderlo, es decir trasmiten un conocimiento experto sin el cual no puede entenderse el texto: credit rating; sovereign downgrades; investment grades; speculative territory; macroeconomic outlook; market confidence; recession; fiscal consolidation; exposure; bank funding costs; long-term yields; fiscal discipline; procyclical; bail-out costs.

Hemos llevado a cabo un estudio del tratamiento lexicográfico de cada una de los términos anteriores en tres tipos de diccionarios de Internet gratuitos: (i) un diccionario especializado (BusinessDictionary.com); un agregador de diccionarios (Dictionary.com); (iii) un diccionario bilingüe (Reverso). El análisis nos confirma la usabilidad de estos diccionarios al ofrecernos definiciones y equivalentes conceptualmente válidos, con niveles de complejidad diferentes, presentes en artículos lexicográficos con referencias cruzadas, ejemplos, información gramatical, frases y colocaciones y otros elementos lexicográficos adecuados. Por ejemplo en (5) mostramos las definiciones y equivalente de uno de estos términos: esta información es suficiente y adecuada si alguien buscara ayuda al leer el texto antes mencionado:

\section{Credit rating}

calificación crediticia

Evaluation of the timely repayment ability of an individual, firm, or debt security (such as a bond). Credit rating is built up on the basis of the (1) credit history, (2) present financial position, and the (3) likely future income. Credit reporting agencies, such as the US firm Dun \& Bradstreet, collect, store, analyze, summarize, and sell such information. Also called debt rating.

an evaluation of the creditworthiness of an individual or business enterprise

An evaluation of the financial trustworthiness of an individual or business enterprise

a classification of credit risk based on investigation of a customer»s or potential customer»s financial resources, prior payment pattern, and personal history or degree of personal responsibility for debts incurred.

Ejemplo 5: credit rating en varios diccionarios de Internet.

La relación entre diccionario pedagógico y recepción de un texto está muy influenciada por los planteamientos actuales sobre la lexicografía como ciencia independiente, por ejemplo el criterio relacionado con el ofrecimiento de mecanismos de desambiguación del significado que faciliten la lectura del texto. Algunos diccionarios de Internet recientes como el Diccionario Inglés-Español de Contabilidad: Recepción (FuertesOlivera y otros, 2012) incorporan estructuras lexicográficas novedosas que hacen a los mismos diccionarios muy adecuados para llevar a cabo la función de recepción. Este es un diccionario L1-L2-L1-L2, es decir lema en inglés, equivalente en español, definición en inglés y definición en español, que ofrece una gran cantidad de oportunidades desconocidas hasta la fecha. Por ejemplo el diccionario puede usarse para detectar diferencias por razones culturales entre el sistema contable inglés y el español. 
Al consultar el Diccionario Inglés-Español de Contabilidad: Recepción el usuario solo recibe los datos lexicográficos que necesita en esa situación de uso: una definición y un equivalente. Si su nivel de inglés es elevado puede hacer uso de la definición en inglés; por el contrario si su nivel de inglés no es muy alto puede recuperar un equivalente en español y una definición en español. Ese usuario, además, ve facilitada su labor porque los motores de búsqueda y marcadores de polisemia y homonimia con los que va equipado facilitan la desambiguación. Por ejemplo, si el usuario hace una búsqueda de fiscal year recupera dos significados diferentes cuya desambiguación es sencilla gracias al empleo conjunto de números arábigos como marcadores de polisemia, definiciones y equivalentes (ejemplo 6):

\section{Fiscal year}

1. A fiscal year is the tax year, i.e. the twelve-month period for which taxpayers must file a tax return, and for which the government estimates the annual budget.

año fiscal

Un año fiscal es un periodo de 12 meses que se utiliza para calcular los impuestos sobre la renta y los impuestos sobre sociedades, y para el que el gobierno prepara el presupuesto anual.

2. A fiscal year is the accounting period for twelve consecutive months, i.e. the reporting period for which an enterprise prepares its financial statements. Typically, a fiscal year follows the calendar year, but may also run in an alternative twelve-month period, e.g. from April 1 to March 31.

año contable

Un año contable es un periodo de tiempo de 12 meses consecutivos. Coincide generalmente con el año natural, aunque en algunos países el año contable va desde el 1 de abril al 31 de marzo.

Ejemplo 6: fiscal year en el Diccionario Inglés-Español de Contabilidad: Recepción

\section{Conclusión}

El ESP es uno de los lenguajes de especialidad más estudiados en todo el mundo. Este hecho se ha traducido en una escasez de materiales y recursos que ha desembocado en una inviabilidad metodológica: no es viable la puesta en marcha de cursos de ESP con metodología colaborativa, es decir cursos diseñados para ser llevados a la práctica por el trabajo conjunto de un profesor de inglés y un experto en el campo. Si la metodología cooperativa, que se consideraba fundamental en los albores del ESP, no es viable podemos preguntarnos si existe alguna alternativa. En este trabajo hemos defendido que el valor futuro de los cursos de ESP vendrá dado por una reconceptualización metodológica que, en primer lugar, tenga en cuenta la consolidación definitiva del inglés como lingua franca y, en segundo lugar, proceda a dotar de valor añadido a recursos novedosos como Internet que pueden ser considerados una especie de sustituto del experto.

La reconceptualización propuesta defiende que debemos modificar el concepto original de proficiency al tiempo que debemos aceptar variantes nuevas, por ejemplo el 
interlenguaje de los estudiantes, y la ausencia de oferta suficiente para una demanda en aumento. En este marco social hemos propuesto una reconceptualización del concepto de ESP que se basa en el uso combinado de tres enfoques -denominados enfoque funcional, enfoque estilístico y enfoque léxico- que pueden facilitar la mejora docente en este contexto dominado por la presencia de dos vectores que no existían cuando empezó a desarrollarse el ESP: el dominio del inglés como lengua de la comunicación internacional; el desarrollo de Internet. Es por ello que definimos el ESP como un proceso de enseñanza/ aprendizaje en marcha, en el que participan seres humanos que pueden comunicarse presencialmente y no presencialmente, que pueden o no pueden formar parte de una misma cultura, que normalmente son capaces de identificar sus necesidades comunicativas específicas, que necesitan compaginar y hacer compatible el conocimiento experto y la competencia discursiva, y que pueden lograrlo en una situación de enseñanza/aprendizaje que hace uso de los condicionamientos teóricos que sustentan el enfoque funcional, estilístico y léxico.

La reconceptualización del ESP facilita el empleo de materiales docentes sacados de Internet como los diccionarios de Internet cuya utilidad pedagógica se verá incrementada si el usuario del mismo tiene una cultura lexicográfica adecuada. Esto significa la toma en consideración de algunos requisitos generales y otros específicos que han sido descritos en este artículo y que resumimos afirmando que los requisitos generales están relacionados con la situación general de uso, las necesidades del aprendiz, la naturaleza de lo que se quiere aprender y la presencia de estructuras lexicográficas que hayan sido pensadas para favorecer la encontrabilidad, comprensibilidad y usabilidad de los diccionarios. Las características específicas se refieren a cada una de las varias funciones que pueda tener un diccionario, por ejemplo el empleo de un diccionario para mejorar la comprensión lectora de un aprendiz. En nuestra opinión los diccionarios de Internet, tanto los estáticos como los dinámicos, pueden usarse en la situación de uso descrita en este trabajo y contribuyen por ello a la viabilidad del concepto de ESP que hemos defendido en este trabajo.

Agradecimientos: al Ministerio de Economía y Competitividad por los fondos aportados para llevar a cabo esta investigación en el marco del proyecto La Teoría Funcional de la Lexicografía y la Construcción de Diccionarios de Internet en Inglés y en Español en el Campo de la Economía (Ref. FFI2011-22885).

\section{Bibliografía}

Bathia, V. K. (2008). «Genre Analysis, ESP and Professional Practice». English for Specific Purposes 27: 161-174.

Bergenholtz, H. y Tarp, Sven (2002). «Die moderne lexikographische Funktionslehre. Diskussionsbeitrag zu neuen und alten Paradigmen, die Wörterbücher als Gebrauchsgegenstände verstehen». Lexicographica. International Annual for Lexicography 18: 253-263.

- (2003). «Two opposing theories: On H.E. Wiegand»s recent discovery of lexicographic functions». Hermes. Journal of Linguistics 31: 171-196.

BogaARds, PAul (1996). «Dictionaries for Learners of English». International Journal of Lexicography 9: 277-320.BusinessDictionary.com: http://www.businessdictionary.com/ (consulta 15 de febrero de 2012). 
Cowie, A.P. (ed.) (1989). Oxford Advanced Learner's» Dictionary of Current English. Oxford: Oxford University press ( $4^{\text {a }}$ edición).

Dictionary.com: http://dictionary.reference.com/ (consulta: 18 de febrero de 2012).

Elosúa, Marcelino (ed.) (2007). Diccionario LID Empresa y Economía. Madrid: LID (11 edición).

Fuertes-Olivera, Pedro A. (2007) «A Corpus-based View of Lexical Gender in Written Business English». English for Specific Purposes, 26 (4): 219-234.

- (2009). «The Function Theory of Lexicography and Electronic Dictionaries: Wiktionary as a Prototype of Collective Multiple-Language Internet Dictionary», en H. Bergenholtz, S. Nielsen y S. Tarp (eds.), Lexicography at a Crossroads: Dictionaries and Encyclopedias Today, Lexicographica Tools Tomorrow. Berna: Peter Lang, 99-134.

- (ed.) (2010a). Specialised Dictionaries for Learners. Berlin y Nueva York: De Gruyter.

- (2010b). «Lexicography for the Third Millennium: Free Institutional Internet Terminological Dictionaries for Learners», en Pedro A. Fuertes-Olivera (ed.), Specialised Dictionaries for Learners. Berlin y Nueva York: De Gruyter, 193-209.

- (2011). «Equivalent Selection in Specialized e-lexicography: A Case Study with Spanish Accounting Terms». Lexikos 21: 95-119.

- (2012). «On the Usability of Free Internet Dictionaries for Teaching and Learning Business English», en S. Granger y M. Paquot (eds.) Electronic Lexicography. Oxford: Oxford University Press.

Fuertes-Olivera, Pedro A. y Arribas-Baño, A. (2008). Pedagogical Specialised Lexicography. The Representation of Meaning in Business and Spanish Business Dictionaries. Amsterdam/Philadelphia: John Benjamins.

Fuertes-Olivera, Pedro A. y Bergenholtz, H. (eds.) (2011) e-Lexicography: The Internet, Digital Initiatives and Lexicography. Londres y Nueva York: Continuum.

Fuertes-Olivera, Pedro A. y Nielsen, Sandro (2011) «The Dynamics of Terms in Accounting: what the Construction of the Accounting Dictionaries Reveals about Metaphorical Terms in Culture-bound Subject Fields, en R. Temmermany M. Van Campenhoudt (eds.), The Dynamics of Terms in Specialized Communication. An Interdisciplinary Perspective. Special Issue of Terminology. International Journal of Applied Issues in Specialized Communication 17(1): 157-180.

- (2012). «Online Dictionaries for Assisting Translators of LSP Texts: The Accounting Dictionaries». International Journal of Lexicography 25. doi: 10.1093/ijl/ecr034.

Fuertes-Olivera, Pedro A. \& Beatriz Pérez Cabello de Alba (2012). «Online Dictionaries and the Teaching/Learning of English in the Expanding Circle». International Journal of English Studies.

Fuertes-Olivera, Pedro A., Henning Bergenholtz, Sandro Nielsen, Pablo Gordo Gómez, Lise Mourier, Marta Niño Amo, Ángel de los Ríos Rodicio, Ángeles Sastre Ruano, Sven Tarp, Marisol Velasco Sacristán. (2012). Diccionario Inglés-Español de Contabilidad: Recepción. Base de Datos y Diseño: Richard Almind y Jesper Skovgård Nielsen. Odense: Lemma.com . ISBN: 978-84-615-6770-6.

KaAleP, Heiki-JAAn y MikK, JAAN (2008). «Creating Specialised Dictionaries for Foreign Language Learners: A Case Study». International Journal of Lexicography 21(4): 369-394.

Koyalan, Aylin y Simon Mumford (2011). «Changes to English as an Additional Language Writers» Research articles: From spoken to written register». English for Specific Purposes 30: 113-123.

Ljosland, Ragnhild (2011). «English as an Academic Lingua Franca: Language Policies and Multicultural Practices in a Norwegian University». Journal of Pragmatics 43: 991-1004. 
Lozano Irueste, José María (2005). Diccionario Bilingüe de Economía y Empresa InglésEspañol, Español-Inglés. Madrid: Ariel. (7 $7^{\mathrm{a}}$ edición).

Munby, John (1978). Communicative Syllabus Design. London: Cambridge UNiversity Press.

Parkinson, D. (con la asistencia de J. Noble (2006). Oxford Business English Dictionary for Learners of English. Oxford: Oxford University Press.

Pujol, Didac, Corrius, Montse y Masnou, Joan (2006) «Print Deferred Bilingualised Dictionaries and Their Implications for Effective Language Learning. A New Approach to Pedagogical Lexicography.» International Journal of Lexicography 19(2): 197-215.

Reverso: http://www.reverso.net/text_translation.aspx?lang=ES (consulta: 18 de febrero de 2012).

Rogerson-Revell, P. (2008). Participation and Performance in International Business Meetings. English for Specific Purposes 27: 338-360.

Rollin, Nicholas y Styles Carvaja, Carol (eds.) (2008). Gran Diccionario Oxford EspañolInglés/Inglés-Español. Oxford: Oxford University Press (4a edición).

Rundell, Michael (1996). «Dictionary Use in Production». International Journal of Lexicography 12 (1): 35-53.

- (ed.) (2007). Macmillan English Dictionary for Advanced Learners. Oxford: Macmillan. (2a edición).

SAmAniego Fernández, Eva (2007) La estilística en los lenguajes de especialidad, en E. Alcaraz Varó, J. Mateo Martínez y F. Yus ramos (eds.) Las lenguas profesionales y académicas, 6779. Barcelona. Ariel, 67-79.

SCHOLfield, Phil (1999). «Dictionary Use in Reception». International Journal of Lexicography 12(1): 13-34.

Sinclair, John (1997). Cobuild English Language Dictionary. London: Collins.

Skehan, Peter (1989). Individual Differences in Second-language Learning. London: Edward Arnold.

Smith, Colin (ed.) (1987). Collins Inglés. Diccionario Español-Inglés/English-Spanish.

TARP, Sven (2004) «Basic Problems of Learner's»Lexicography». Lexikos 14: 222-252.

- (2008) Lexicography in the Borderland between Knowledge and Non-Knowledge. General Lexicographical Theory with Particular Focus on Learner»s Lexicography. Tübingen: Niemeyer.

Verlinde Serge y Binon, Jean (2009) «Pedagogical Lexicography Revisited», en H. Bergenholtz, S. Nielsen y S. Tarp (eds), Lexicography at a Crossroads. Dictionaries and Enciclopedias Today, Lexicographical Tools Tomorrow, 69-89. Bern: peter Lang.

Zhang, Zuocheng (2007). «Towards an Integrated Approach to Teaching Business English: A Chinese Experience». English for Specific Purposes 26: 399-410. 